\title{
SÍNTESIS, CARACTERIZACIÓN y APLICACIÓN DE ARMAZONES METAL ORGÁNICOS EN LAADSORCIÓN DE DIMETILAMINA
}

\author{
María del Rosario Sun-Kou ${ }^{*}$, Fabiola Bravo Hualpa ${ }^{1}$, Rodrigo Beltrán Suito ${ }^{1}$, \\ Gino Picasso Escobar ${ }^{2}$, Christian Samanamu ${ }^{1}$
}

\begin{abstract}
RESUMEN
Se estudió la adsorción de dimetilamina (DMA) sobre armazones metal orgánicos (MOF). Para ello se realizó la preparación de los MOF empleando la técnica de síntesis solvotérmica. La caracterización de los armazones obtenidos se realizó mediante espectroscopía de reflectancia total atenuada (ATR), espectroscopía de resonancia magnética nuclear $\left({ }^{1} \mathrm{H}-\right.$ $\mathrm{RMN}$ ), difracción de rayos X(DRX), espectroscopía de energía dispersiva de rayos X (EDX) y microscopía electrónica de barrido (SEM). Los resultados de difracción de rayos X mostraron que la estructura de los MOF correspondió a la Dashkovita, cuya fórmula molecular es $\mathrm{Mg}(\mathrm{HCOO})_{2} \cdot 2 \mathrm{H}_{2} \mathrm{O}$. El espectro de ATR indicó que el grupo funcional más importante en los MOF es el grupo carbonilo y el análisis morfológico mostró que las partículas cristalinas de los MOF presentaron una forma hexagonal, formadas por filamentos de alrededor de 7,5-8 $\mu \mathrm{m}$ de longitud.

Los ensayos de adsorción mostraron que el MOF presentó una alta capacidad de adsorción de $\operatorname{DMA}\left(\mathrm{q}_{\mathrm{e}}=307,96 \mathrm{mg} \cdot \mathrm{g}^{-1}\right)$. Los resultados cinéticos se correlacionaron mejor a los modelos de pseudo segundo orden y de Elovich, mientras que la isoterma de adsorción tuvo un mejor ajuste con los modelos de Temkin y de Dubinin-Radushkevich relacionados a procesos preferentemente del tipo químico sobre una superficie heterogénea.
\end{abstract}

Palabras clave: Armazones metal orgánicos, MOF, dimetilamina, adsorción

\section{SYNTHESIS, CHARACTERIZATION AND APPLICATION OF METAL ORGANIC FRAMEWORKS IN THE ADSORPTION OF DIMETHYLAMINE}

\begin{abstract}
This study investigated the removal of dimethylamine (DMA) by an adsorption mechanism using metal-organic frameworks (MOFs). The synthesis of the MOFs was performed by solvothermal methods. The characterization of the MOF obtained was made by attenuated total reflectance spectroscopy (ATR), proton nuclear magnetic resonance spectroscopy $\left({ }^{1} \mathrm{H}\right.$ NMR), X-ray diffraction (XRD), energy dispersive X-ray spectroscopy (EDX) and scanning electron microscopy (SEM). The XRD diffractograms allowed to identify the structure of MOF as Dashkovaite, which has the molecular formula $\mathrm{Mg}(\mathrm{HCOO})_{2} \cdot 2 \mathrm{H}_{2} \mathrm{O}$; while the ATR

1 Departamento de Ciencias-Sección Química. Pontificia Universidad Católica del Perú. Avenida Universitaria 1801 - Lima 32.

2 Laboratorio de Investigación de Fisicoquímica - Facultad de Ciencias. Universidad Nacional de Ingeniería. Avenida Túpac Amaru 210 - Lima 25.

msun@pucp.edu.pe
\end{abstract}


studies revealed the presence of carbonyl group as most important functional group in the MOF structure. The morphological analysis showed that the MOF crystalline particles had a hexagonal shape, formed from filaments of around 7,5-8 microns in length.

Adsorption experiments showed that the MOF had a high adsorption capacity of DMA $\left(\mathrm{q}_{\mathrm{e}}=\right.$ $307,96 \mathrm{mg} \cdot \mathrm{g}^{-1}$ ). The kinetic data were fitted to the pseudo second order equation and the Elovich equation, while the adsorption isotherm was fitted to the Temkin equation and the Dubinin - Radushkevich equation, processes related to chemisorptions preferably on a heterogeneous surface.

Key words: Metal-organic frameworks, MOF, dimethylamine, adsorption.

\section{INTRODUCCIÓN}

Los armazones metal orgánicos (metal organic framework, MOF) son un nuevo tipo de materiales microporosos que se forman al conectar especies orgánicas y metales de transición por medio de enlaces fuertes (síntesis reticular) ${ }^{1}$. Las especies orgánicas son carboxilatos orgánicos politópicos (u otras moléculas similares con carga negativa) que al enlazarse a los metales de transición producen estructuras porosas en forma de armazones cristalinos ${ }^{1}$. El tamaño específico de poro de estos armazones está determinado por la estructura misma del material, la cual varía según la naturaleza de las especies orgánicas. Por otro lado, los metales de transición actúan como nodos en la estructura del armazón, cuya geometría de coordinación está determinada por la naturaleza del metal ${ }^{1}$. Además, las especies orgánicas con diversos grupos funcionales actúan como puentes de extensión que unen los diferentes nodos metálicos, permitiendo que la estructura se extienda formando una red ${ }^{2}$. Esto ofrece una combinación sinérgica de propiedades que pueden ser dirigidas hacia múltiples aplicaciones, entre las que destacan el almacenamiento de combustibles (hidrógeno y metano), la captura de dióxido de carbono, aplicaciones en adsorción y catálisis ${ }^{2}$.

Existen diferentes métodos de preparación de los MOF, entre las que destaca la síntesis solvotérmica ${ }^{1}$, en la cual el crecimiento de los cristales (o microcristales) de los MOF se realiza en soluciones de solventes orgánicos a altas temperaturas. Esta solución homogénea del metal de transición y el ligando orgánico se mantiene en maduración por el tiempo necesario para precipitar los MOF. En esta investigación se aplicó este método de síntesis a altas temperaturas empleando dimetilformamida (DMF) como solvente. Una vez sintetizados, los MOF fueron utilizados como adsorbentes para la retención de la dimetilamina (DMA).

La dimetilamina es un compuesto que tiene un variado uso en diversas industrias: textiles (para mejorar la fuerza de tensión), farmacéuticas (para sintetizar anestésicos, tranquilizantes, antiestamínicos y otros) y agroquímicas (para inhibir la nitrificación de suelos, sintetizar herbicidas y otros $)^{3}$. La importancia de su remoción en los efluentes industriales se genera a consecuencia de que el tratamiento que se da a las aguas residuales (uso de cloraminas, cloro $u$ ozono en la etapa desinfección) puede transformar DMA en N-nitrosodimetilamina $(\mathrm{NDMA})^{4}$, la cual se encuentra en la lista de los contaminantes prioritarios de la Agencia para la Protección Ambiental (EPA) ${ }^{4}$ de EE.UU. El límite máximo permisible de la NDMA para agua potable está en el intervalo de $9-10 \mathrm{ng} / \mathrm{L}^{4}$. Estos valores son bastante bajos con respecto al de la dimetilamina, cuyo límite máximo permisible establecido por la Administración de Seguridad y Salud (OSHA) es $10 \mathrm{mg} / \mathrm{L}^{5}$. Esto revela que la reacción de oxidación de la dimetilamina genera un gran incremento en la toxicidad debido a su transformación en NDMA y en esto radica la importancia de su remoción mediante la adsorción de la DMA sobre los MOF. 


\section{PARTE EXPERIMENTAL}

La síntesis de los MOF se realizó mezclando $30 \mathrm{~mL}$ de dimetilformamida $\left(\mathrm{C}_{3} \mathrm{H}_{7} \mathrm{NO}\right.$, Merck, 99,9\%), 2,3mL de ácido fórmico $\left(\mathrm{CH}_{2} \mathrm{O}_{2}\right.$, Sigma-Aldrich, 85\%) y entre 7,7 a 7,9 $\mathrm{g}$ de nitrato de magnesio hexahidratado $\left(\mathrm{Mg}\left(\mathrm{NO}_{3}\right)_{2} 6 \mathrm{H}_{2} \mathrm{O}\right.$, Merck, 99\%). Esta mezcla fue colocada dentro de un tubo Schlenk, el cual se cerró herméticamente y se colocó dentro de un baño de silicona caliente $\left(96 \mathrm{a} 110^{\circ} \mathrm{C}\right)$ y se mantuvo en agitación por un tiempo entre $40 \mathrm{a} 45 \mathrm{~h}$. Al culminar el tiempo de reacción, los cristales blancos depositados fueron filtrados al vacío y sometidos a un tratamiento térmico en una estufa a $120^{\circ} \mathrm{C}$ por 48 horas. Posteriormente estos cristales fueron transferidos a viales, rotulados con el término MOFX (X indica el número de ensayo realizado) y mantenidos en un desecador.

La caracterización estructural se realizó por difracción de rayos $\mathrm{X}(\mathrm{DRX})$ en un difractómetro marca Bruker, modelo D8-FOCUS con un detector de centelleo $\left(\lambda_{\mathrm{Cu}-\mathrm{K \alpha} 1}=1,5406 \AA\right)$. El equipo se operó a $40 \mathrm{kV}$ y $40 \mathrm{~mA}$, y los datos se colectaron en el rango de $2 \theta=10^{\circ}-80^{\circ}$. Las fases cristalinas fueron identificadas por comparación con los espectros DRX de la base de datos ICDD-2007.

La determinación de los grupos funcionales se llevó a cabo mediante reflectancia total atenuada (ATR) usando el accesorio Universal ATR del espectrómetro Spectrum 100 FT-IR y los espectros se registraron en el rango de $4000-400 \mathrm{~cm}^{-1}$. Estas pruebas se complementaron con los análisis por espectroscopía de resonancia magnética nuclear ( $\left.{ }^{1} \mathrm{H}-\mathrm{RMN}\right)$, los cuales se realizaron en un espectrómetro de Resonancia Magnética Nuclear marca Bruker de $300 \mathrm{MHz}$ con una consola Avance III 300. Se utilizó $\mathrm{D}_{2} \mathrm{O}$ (99,9\%, Merck) como solvente.

La determinación de la composición elemental de las muestras se realizó mediante la espectroscopía de energía dispersiva de rayos X (EDX) y el análisis morfológico se llevó a cabo a través de la microscopía electrónica de barrido (SEM). Ambos ensayos se realizaron en un microscopio electrónico de barrido FEI Quanta 200.

Los ensayos cinéticos se llevaron a cabo en un sistema "batch". Para ello, en diferentes viales se mezcló 15 mg de MOF y 10mL de solución de DMA(500 ppm, sin ajuste de pH). La mezcla fue colocada sobre un agitador múltiple (VELP Scientifica Multistirrer 15) a temperatura ambiente y se dejó en contacto por un determinado tiempo entre $30 \mathrm{~min}$ a $6 \mathrm{~h}$. Luego la mezcla fue filtrada al vacío y se cuantificó la cantidad de DMA en la solución remanente usando un espectrofotómetro de UV Visible Agilent 8453. Debido a que la DMA no se puede detectar directamente por UV-visible, al no presentar grupos cromóforos en su estructura, se realizó una derivatización previa a la medición usando 1,3-dinitrobenceno (DNB) para formar un complejo donador-aceptor de electrones (electron-donor acceptor ${ }^{6}$, EDA por sus siglas en inglés), el cual adquiría un color violeta en presencia de acetona y a $\mathrm{pH}=12$. Las mediciones se realizaron a una longitud de onda $\lambda=555 \mathrm{~nm}$ luego de haber transcurrido un minuto de haberse preparado el complejo.

La curva cinética de adsorción se obtuvo al graficar la capacidad de adsorción en el tiempo t $\left(\mathrm{q}_{\mathrm{t}}, \mathrm{mg} \cdot \mathrm{g}^{-1}\right)$ versus el tiempo $(\mathrm{t}, \mathrm{min})$; mientras que la isoterma de adsorción se obtuvo al graficar la capacidad de adsorción en el equilibrio $\left(\mathrm{q}_{\mathrm{e}}, \mathrm{mg} \cdot \mathrm{g}^{-1}\right)$ versus la concentración del adsorbato en el equilibrio $\left(\mathrm{C}_{\mathrm{e}}, \mathrm{mg} \cdot \mathrm{L}^{-1}\right)$. El cálculo de la capacidad de adsorción $\left(\mathrm{q}_{\mathrm{t}}\right)$ se realizó mediante la ecuación 1.

$$
q_{t}=\frac{V\left(C_{0}-C_{t}\right)}{m} \quad \text { Ecuación } 1
$$


Donde: $\mathrm{C}_{\mathrm{o}}$ es la concentración inicial del adsorbato $\left(\mathrm{mg} \mathrm{L}^{-1}\right), \mathrm{C}_{\mathrm{t}}$ es la concentración del adsorbato en el tiempo " $\mathrm{t}$ " (mg. $\left.\mathrm{L}^{-1}\right), \mathrm{V}$ es el volumen de la solución en litros, $\mathrm{m}$ es la masa de adsorbente en gramos y $\mathrm{q}_{\mathrm{t}}\left(\mathrm{mg} \cdot \mathrm{g}^{-1}\right)$ representa la capacidad de adsorción en el tiempo " $\mathrm{t}$ ".

Para la isoterma de adsorción, se usó soluciones de DMA con concentraciones iniciales entre 100 a $1600 \mathrm{ppm}$ y el tiempo de contacto fue de 4 horas para cada punto de la isoterma. Se trabajó a temperatura ambiente y en condiciones de equilibrio. El valor de $\mathrm{q}_{\mathrm{e}}$ se obtuvo mediante una ecuación similar a la ecuación 1 , reemplazando los términos de $\mathrm{q}_{\mathrm{t}}$ por $\mathrm{q}_{\mathrm{e}} \mathrm{y}$ el de $\mathrm{C}_{\mathrm{t}}$ por $\mathrm{C}_{\mathrm{e}}$.

Para la correlación de los resultados cinéticos de adsorción se utilizó los modelos de pseudo primer y segundo orden, el de Elovich y el de difusividad intraparticular. En el caso de los resultados experimentales de la isoterma de adsorción, se empleó los modelos linealizados de Langmuir, Temkin, Freundlich y Dubinin-Radushkevich. Además del coeficiente correlación lineal $\left(\mathrm{R}^{2}\right)$ de cada modelo, se utilizó el test de Chi cuadrado no lineal $\left(\chi^{2}\right)$, que se obtiene mediante la ecuación 2 . El valor de $\chi^{2}$ permite una mejor evaluación de la precisión de los modelos ya que ofrece una comparación estadística de todas las curvas bajo la misma abscisa y ordenada. De acuerdo a este test, se tiene que los valores de $\chi^{2}$ más cercanos a cero indicarían que el modelo se ajusta mejor a los valores experimentales ${ }^{7}$

$$
\chi^{2}=\sum \frac{\left(\mathrm{q}_{\mathrm{e}}-\mathrm{q}_{\mathrm{em}}\right)^{2}}{\mathrm{q}_{\mathrm{em}}}
$$

Ecuación 2

Donde: $\chi^{2}$ es el Chi cuadrado, $\mathrm{q}_{\mathrm{e}}\left(\mathrm{mg} \cdot \mathrm{g}^{-1}\right)$ es la capacidad de adsorción experimental y $\mathrm{q}_{\mathrm{em}}\left(\mathrm{mg} \cdot \mathrm{g}^{-1}\right)$ es la capacidad de adsorción según modelo de isoterma.

\section{RESULTADOS Y DISCUSIÓN}

Se realizó la síntesis de los MOF de acuerdo al procedimiento descrito anteriormente. Las mejores condiciones de preparación y de rendimiento de los MOF se muestran en la tabla 1. Para el cálculo del rendimiento de cada ensayo se tomó en cuenta un peso molecular de 172,49 g.mol ${ }^{-1}$ que corresponde a $\mathrm{Mg}(\mathrm{HCOO})_{2} \cdot 2 \mathrm{H}_{2} \mathrm{O}$, el cual representa la fórmula molecular del MOF (verificada por ensayos de DRX). La síntesis del MOF se esquematiza a través de la ecuación 3.

$$
\mathrm{Mg}\left(\mathrm{NO}_{3}\right)_{2} \cdot 6 \mathrm{H}_{2} \mathrm{O}+2 \mathrm{HCOOH} \rightarrow \mathrm{Mg}(\mathrm{HCOO})_{2} \cdot 2 \mathrm{H}_{2} \mathrm{O}+4 \mathrm{H}_{2} \mathrm{O}+2 \mathrm{HNO}_{3} \quad \text { Ecuación } 3
$$


Tabla 1. Condiciones de reacción y valores de rendimiento obtenidos en la síntesis de los armazones organometálicos (MOF).

\begin{tabular}{cccccc}
\hline Rótulo & $\begin{array}{c}\text { Masa de } \\
\text { nitrato }(\mathbf{g})\end{array}$ & $\begin{array}{c}\text { Volumen } \\
\text { de ácido } \\
(\mathbf{m L})\end{array}$ & $\begin{array}{c}\text { Masa } \\
\text { obtenida de } \\
\text { MOF }(\mathbf{g})\end{array}$ & Rendimiento & $\begin{array}{c}\text { Temperatura } \\
\text { de síntesis } \\
\left({ }^{\circ} \mathbf{C}\right)\end{array}$ \\
\hline MOF8 & 7,7648 & 2,3 & 3,5560 & $68 \%$ & 105 \\
MOF13a & 7,7231 & 2,3 & 1,9863 & $38 \%$ & 105 \\
MOF13b & 7,7262 & 2,3 & 2,0841 & $40 \%$ & 105 \\
MOF14 & 7,7526 & 2,3 & 3,4850 & $67 \%$ & 105 \\
MOF15a & 7,7278 & 2,3 & 3,2985 & $64 \%$ & 105 \\
MOF15b & 7,7125 & 2,3 & 3,2985 & $64 \%$ & 105 \\
\hline
\end{tabular}

En la nomenclatura, los términos a y b corresponden a ensayos realizados por duplicado.

El análisis por difracción de rayos X (DRX) permitió identificar la estructura de los MOF. En la figura 1 se observa el difractograma de rayos X de la muestra MOF13a, el cual se comparó con la ficha JCPDS53-1187 obtenida de la base de datos ICDD-2007 ${ }^{8}$. Como se puede apreciar, los picos característicos del MOF13a coinciden en la mayoría de los casos con la ficha de la Dashkovaita correspondiente a la estructura $\alpha-\mathrm{Mg}\left(\mathrm{HCO}_{2}\right)_{2} \cdot 2 \mathrm{H}_{2} \mathrm{O}$. Los picos que aparecen a valores de $2 \theta$ de $13,54^{\circ} ; 14,19^{\circ} ; 14,47^{\circ}$ y $14,98^{\circ}$ corresponderían a la formación de una segunda fase cristalina no identificada por la base de datos ICDD ${ }^{8}$.

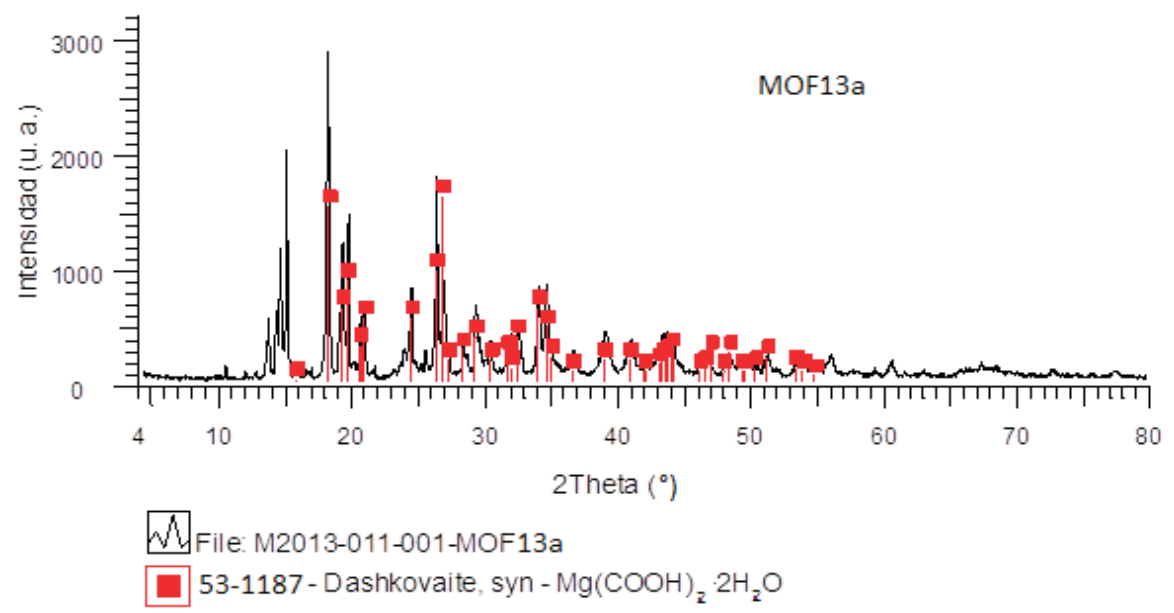

Figura 1. Difractograma de rayos X de la muestra MOF13a.

Los espectros de ATR experimentales se compararon con las reportadas en el estudio realizado por Rood y col. ${ }^{9}$, quienes realizaron la preparación de un compuesto organometálico muy parecido a los MOF sintetizado en este estudio. El análisis de los espectros ATR de los MOF permitió identificar tres zonas de absorción comunes. Las señales comprendidas entre 1670 y $1600 \mathrm{~cm}^{-1}$ fueron atribuidas al grupo carbonilo perteneciente al ligando formiato. Mientras que 
las señales alrededor de 1404 y $1347 \mathrm{~cm}^{-1}$ se asociaron a vibraciones de tensión -C-O-C y a vibraciones de flexión del O-H (carboxílico), respectivamente. El espectro ATR de la muestra MOF8 (figura 2) muestra que el principal grupo funcional en la estructura de los MOF es el grupo carbonilo.

Los análisis por ${ }^{1} \mathrm{H}-\mathrm{RMN}$ (tabla 2) permitieron verificar la presencia de grupos formiato en la estructura y comprobar que no hubiera moléculas de DMF o DMA en las muestras después del tratamiento térmico. Las señales a $\delta 8,25 \mathrm{ppm}$ (singulete) y a $\delta 4,70 \mathrm{ppm}$ fueron asociadas al grupo formiato y al $\mathrm{D}_{2} \mathrm{O}$, respectivamente ${ }^{9}$. Las señales a $\delta 2,8 \mathrm{ppm}$ y $\delta 2,7 \mathrm{ppm}$ correspondieron a los protones metílicos del DMF; la señal a $\delta 7,7 \mathrm{ppm}$ se asoció al protón del grupo amida del DMF y la señal a $\delta 2,5 \mathrm{ppm}$ se asoció a los protones metílicos DMA.

Tabla 2. Asignación de señales de los espectros ${ }^{1} \mathrm{H}-\mathrm{RMN}$ de los MOF.

\begin{tabular}{|c|c|c|c|c|c|c|}
\hline \multirow{2}{*}{ Muestra } & \multicolumn{6}{|c|}{ Átomo asignado } \\
\hline & $\mathrm{O}_{2} \mathrm{CH}$ & $\mathrm{HON}\left(\mathrm{CH}_{3}\right)_{2}$ & $\mathrm{D}_{2} \mathrm{O}$ & $\mathrm{HON}\left(\mathrm{CH}_{3}\right)_{2}$ & $\mathrm{HON}\left(\mathrm{CH}_{3}\right)_{2}$ & $\mathrm{HNCH}_{3}$ \\
\hline Ref. $^{9}$ & 8,44 & 7,92 & 4,70 & 3,00 & 2,84 & 2,50 \\
\hline MOF8 & 8,28 & - & 4,70 & - & - & 2,55 \\
\hline MOF13a & 8,27 & - & 4,70 & - & - & 2,55 \\
\hline MOF13b & 8,28 & - & 4,70 & - & - & - \\
\hline MOF14 & 8,28 & - & 4,70 & - & - & 2,55 \\
\hline MOF15a & 8,27 & 7,76 & 4,69 & 2,85 & 2,69 & 2,55 \\
\hline MOF15b & 8,28 & 7,77 & 4,70 & 2,85 & 2,70 & 2,56 \\
\hline
\end{tabular}

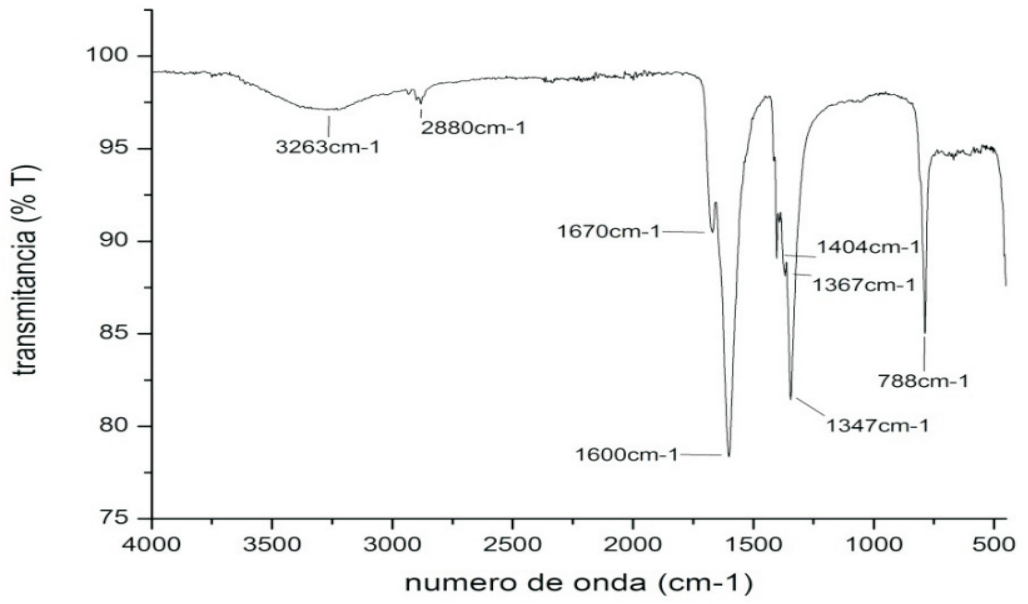

Figura 2. Espectro ATR de la muestra MOF8.

En la micrografía SEM del MOF13a con una ampliación de 3000X (figura 3, centro), se observa la formación de un cristal con forma casi hexagonal, el cual está conformado a su vez por pequeñas fibras de aproximadamente $7,4 \mu \mathrm{m}$ de longitud. En la figura 3 (lado derecho) se muestra la composición elemental del MOF13a obtenida mediante el análisis EDX total que fue de $\mathrm{C}(27,08 \%), \mathrm{O}(55,21 \%)$ y $\mathrm{Mg}(17,71 \%)$. 


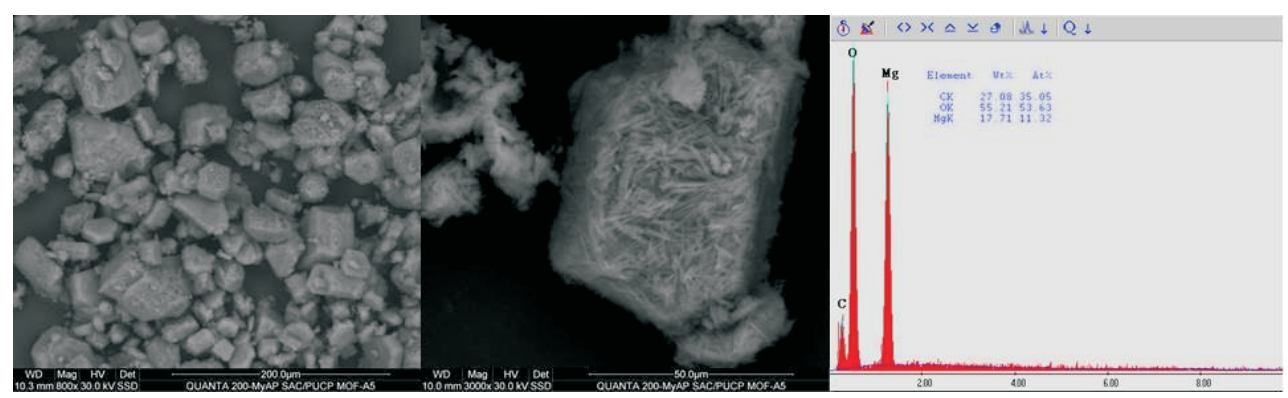

Figura 3. Micrografías SEMa 800X y 3000 X, y análisis EDX total de la muestra MOF13a.

En la micrografía SEM de la muestra MOF13b (figura 4, lado izquierdo) con 1500X de aumento se observa la formación de partículas cristalinas hexagonales más regulares que las del MOF13a, las cuales están formadas por filamentos alargados de aproximadamente $8 \mu \mathrm{m}$ de longitud (figura 4, centro). En esta figura 4 (lado derecho) se muestra la composición elemental del MOF13b la cual fue de C (25,61\%), O (52,41\%) y Mg (21,98\%). Los ensayos realizados con ambas muestras evidenciaron que el proceso de síntesis es reproducible y hay homogeneidad en toda la muestra sintetizada.
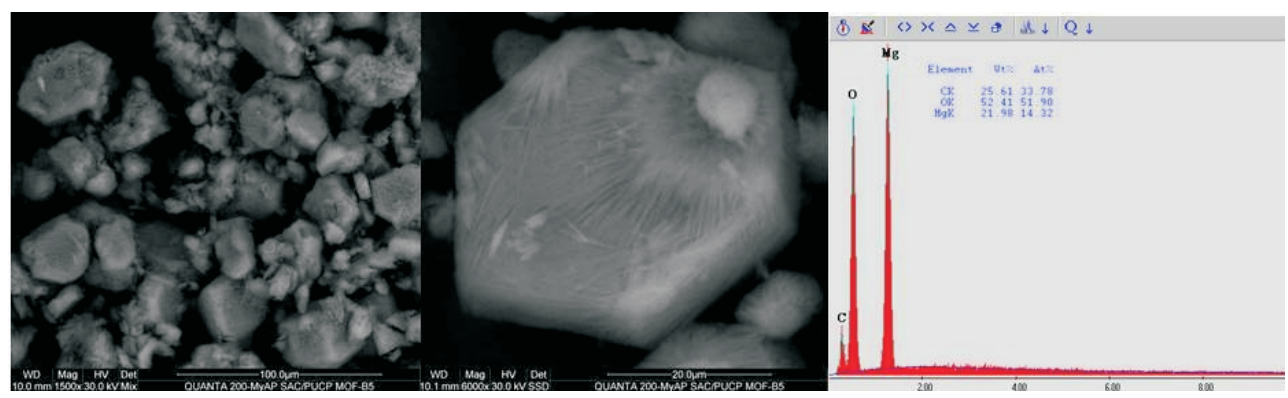

Figura 4. Micrografías SEM a 1500X y 6000X, y análisis EDX total de la muestra MOF13b.

La curva cinética experimental obtenida para el MOF13a (figura 5a) indica que para una concentración inicial de $500 \mathrm{mg} \cdot \mathrm{g}^{-1}$ de DMA, la adsorción es rápida sólo en los primeros instantes de contacto y luego el proceso se vuelve más lento hasta alcanzar las condiciones de equilibrio $\left(\mathrm{q}_{\mathrm{e}}=307,96 \mathrm{mg} \cdot \mathrm{g}^{-1}\right)$. Por otro lado, la isoterma de adsorción experimental (figura 5b) es del tipo $\mathrm{S}$ de acuerdo a la clasificación Giles ${ }^{10}$ que se evidencia por tener una forma ligeramente convexa en la parte inicial de la adsorción, debido posiblemente, a la resistencia ocasionada por el trasporte de masa o difusión en la interfase sólido-líquido. El valor de $\mathrm{q}_{\mathrm{e}} \mathrm{se}$ incrementa lentamente para $\mathrm{C}_{\mathrm{e}}$ bajos y luego aumenta rápidamente hasta un valor de 538,1 mg. $\mathrm{g}^{-1}$; el proceso aparentemente tiende a estabilizarse y después tiende a aumentar para $\mathrm{C}_{\mathrm{e}}$ altos, lo que permite elucidar que el MOF no presenta claramente un límite de su capacidad de adsorción. 

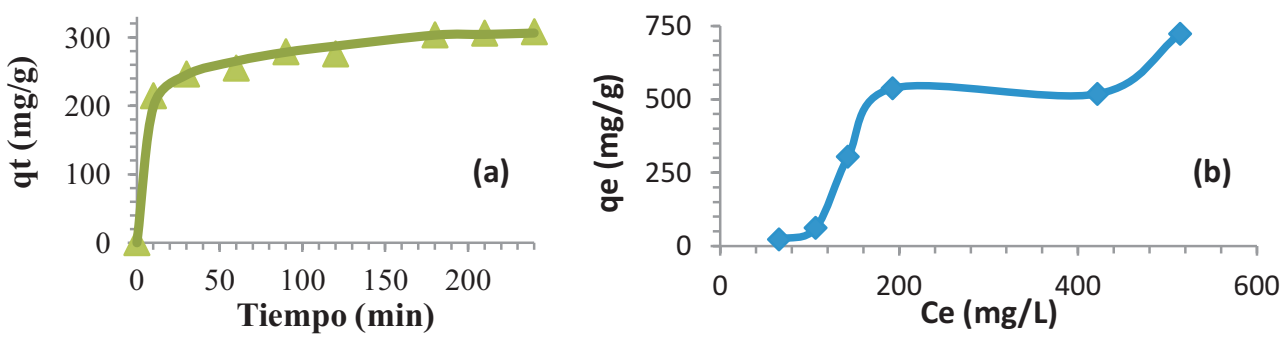

Figura 5. (a) Curva cinética de adsorción experimental para elMOF13a. (b) Isoterma de adsorción experimental para el MOF13a.

El estudio cinético de adsorción se realizó por medio de la aplicación de cuatro modelos cinéticos: pseudo primer y segundo orden, Elovich y difusividad intraparticular ${ }^{11}$. La tabla 3 muestra en forma comparativa los coeficientes de correlación $\left(\mathrm{R}^{2}\right)$, los valores de los parámetros de cada modelo.

Tabla 3. Parámetros y coeficientes de correlación de los modelos cinéticosde pseudo primer orden, pseudo segundo orden, Elovich y difusividad intraparticular.

\begin{tabular}{|c|c|c|c|c|c|}
\hline \multicolumn{6}{|c|}{ PSEUDO PRIMER ORDEN } \\
\hline $\mathrm{q}_{\mathrm{em}}\left(\mathrm{mg} \cdot \mathrm{g}^{-1}\right)$ & \multicolumn{2}{|c|}{$\mathrm{k}_{1}\left(\min ^{-1}\right)$} & \multicolumn{2}{|l|}{$\mathrm{R}^{2}$} & $\chi^{2}$ \\
\hline 178,76 & \multicolumn{2}{|c|}{$2,00 \times 10^{-2}$} & \multicolumn{2}{|c|}{0,931} & $2,07 \times 10^{3}$ \\
\hline \multicolumn{6}{|c|}{ PSEUDO SEGUNDO ORDEN } \\
\hline $\mathrm{q}_{\mathrm{em}}\left(\mathrm{mg} \cdot \mathrm{g}^{-1}\right)$ & \multicolumn{2}{|c|}{$\mathrm{k}_{2}\left(\mathrm{~g} \cdot \mathrm{mg}^{-1} \min ^{-1}\right)$} & \multicolumn{2}{|c|}{$\mathrm{R}^{2}$} & $\chi^{2}$ \\
\hline 319,081 & \multicolumn{2}{|c|}{$2,85 \times 10^{-4}$} & \multicolumn{2}{|c|}{0,997} & 28,340 \\
\hline \multicolumn{6}{|c|}{ ELOVICH } \\
\hline$(1 / b) \operatorname{Ln}(a b)\left(m g \cdot g^{-1}\right)$ & 1/b (mg.g $\left.{ }^{-1}\right)$ & $\mathrm{a}$ & $\mathrm{b}$ & $\mathrm{R}^{2}$ & $\chi^{2}$ \\
\hline 142,805 & 29,474 & $3,75 \times 10^{3}$ & $3,39 \times 10^{-2}$ & 0,955 & 1,142 \\
\hline \multicolumn{6}{|c|}{ DIFUSIVIDAD INTRAPARTICULAR } \\
\hline $\mathrm{k}_{\mathrm{p} 1}\left(\mathrm{mg} \cdot \mathrm{g}^{-1} \min ^{-0.5}\right) \quad \mathrm{k}_{\mathrm{p} 2}\left(\mathrm{mg} \cdot \mathrm{g}^{-1} \min ^{-0.5}\right)$ & $\mathrm{k}_{\mathrm{p} 3}\left(\mathrm{mg} \cdot \mathrm{g}^{-1} \min ^{-0.5}\right)$ & $\mathrm{R}_{1}^{2}$ & $\mathrm{R}_{2}^{2}$ & $\mathrm{R}_{3}^{2}$ & $\chi^{2}$ \\
\hline 67,834 & 2,214 & 1 & 0,943 & 0,998 & 0,878 \\
\hline
\end{tabular}

El modelo de pseudo primer orden se descartó debido a la baja exactitud en la predicción de la cinética experimental $\left(\mathrm{R}^{2}\right)$ y por presentar una capacidad máxima de adsorción $\left(\mathrm{q}_{\mathrm{em}}=178,76\right.$ mg. $\left.\mathrm{g}^{-1}\right)$ lejana a la encontrada experimentalmente $\left(\mathrm{q}_{\mathrm{e}}=307,96 \mathrm{mg} \cdot \mathrm{g}^{-1}\right.$ a partir de una concentración inicial de $500 \mathrm{mg} \cdot \mathrm{g}^{-1}$ de DMA). Al comparar los valores de chi cuadrado $\left(\chi^{2}\right)$, se estableció que el modelo de Elovich predecía con buena exactitud los resultados experimentales al tener un bajo valor de $\chi^{2}$, seguido del modelo de pseudo segundo orden (tabla 3 ). 
La buena correlación lineal $\left(\mathrm{R}^{2}\right)$ de los modelos de pseudo segundo orden y de Elovich permitió elucidar que el proceso de adsorción es el resultado de una interacción preferentemente del tipo químico (quimisorción) ${ }^{12}$. Además, el bajo valor de la constante de velocidad $\left(\mathrm{k}_{2}\right)$ del modelo de pseudo segundo orden indicaría que la adsorción en general es lenta, como se observa en la forma de la curva cinética (figura 6), que presenta un ascenso lento hasta alcanzar las condiciones de equilibrio después de 4 horas de contacto. En el caso del modelo de Elovich, tanto el número de sitios disponibles para adsorción $(1 / \mathrm{b})$ y la cantidad adsorbida en el tiempo de $\mathrm{t}=1 \mathrm{~min}(1 / \mathrm{b} \operatorname{Ln}(\mathrm{ab}))$, son valores altos, lo que estaría en concordancia con la alta afinidad del MOF por la DMA y la alta capacidad de adsorción observada experimentalmente $\left(\mathrm{q}_{\mathrm{e}}=307,96 \mathrm{mg} \cdot \mathrm{g}^{-1}\right)$.

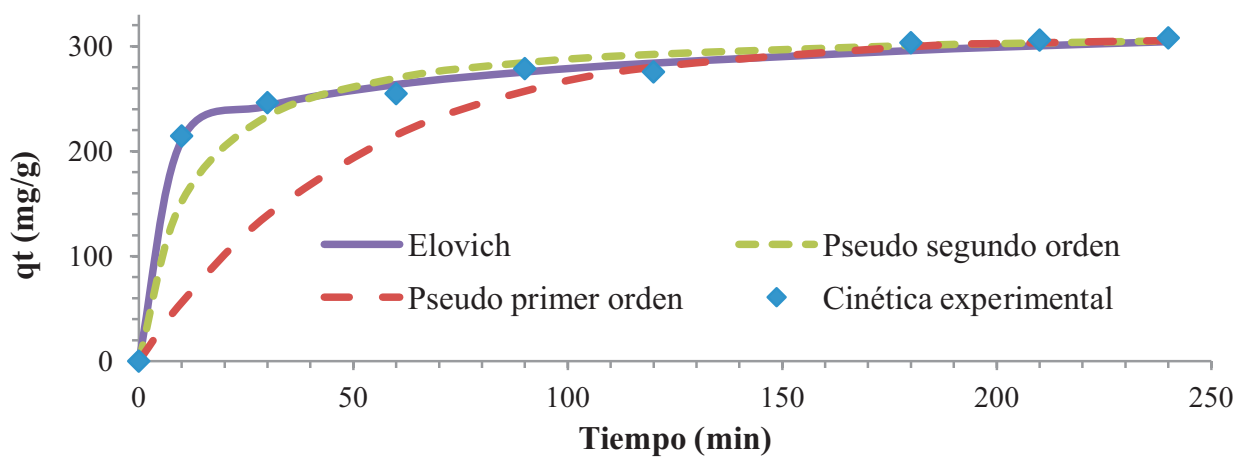

Figura 6. Comparación de la cinética de adsorción experimental con los modelos cinéticos para la muestra MOF13a.

Al aplicar el modelo de difusividad intraparticular ${ }^{11}$, se identificaron tres etapas en el proceso de adsorción (figura 7). Se observa al inicio una porción lineal con valores de $\mathrm{t}^{0,5}$ entre 0 y 3 min, que de acuerdo con el modelo estaría asociado con la difusión del adsorbato a través de la interfase líquido-sólido. Este comportamiento lineal indicaría que este transporte de masa ocurre rápidamente y no es una etapa limitante en el proceso. La segunda etapa a valores de $\mathrm{t}^{0,5}$ entre 3 y $13 \mathrm{~min}$, se asocia con el transporte del adsorbato a través de los poros del adsorbente (difusión interna) y la tercera etapa por una velocidad de difusión intraparticular en reducción hasta alcanzar el estado equilibrio. La tabla 3 muestra las constantes de velocidad de la primera $\left(\mathrm{kp}_{1}\right)$, segunda $\left(\mathrm{kp}_{2}\right)$ y tercera $\left(\mathrm{kp}_{3}\right)$ etapa. Siendo la primera constante la de más alto valor. Por el comportamiento observado en la adsorción de DMA, se podría elucidar que existe una influencia significativa del transporte de masa en el proceso de adsorción 


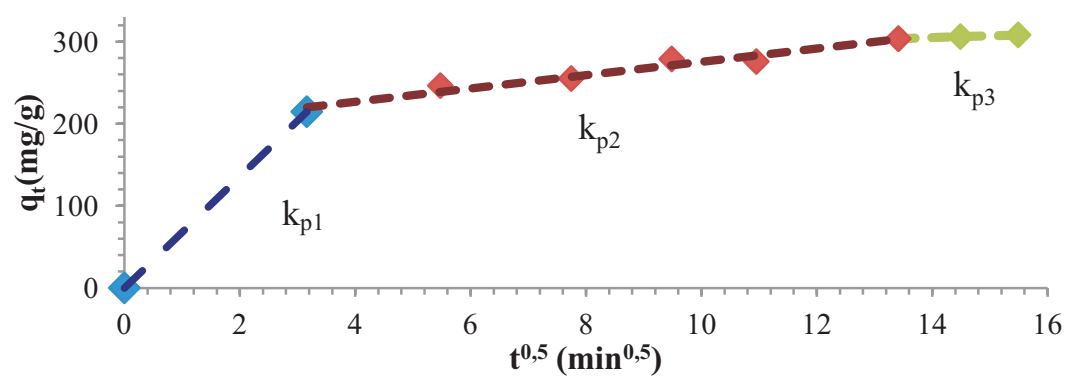

Figura 7. Ajustes lineales para la cinética de adsorción mediante el modelo de difusividad intraparticular.

La correlación de los resultados experimentales de la adsorción de la DMA sobre el MOF se realizó empleando los modelos de isotermas ${ }^{11}$ de Langmuir, Freundlich, DubininRadushkevich (D-R) y Temkin. La tabla 4 muestra un resumen de los $\mathrm{R}^{2}$ y los parámetros de cada modelo aplicado. Se puede observar por los valores de $\mathrm{R}^{2} \mathrm{y} \chi^{2}$ que ninguno de los modelos muestra una alta correlación lineal con los valores experimentales. El mayor ajuste se obtuvo con los modelos de Temkin y D-R. En la figura 8 se observa las gráficas comparativas de los cuatro modelos con los resultados experimentales.

El modelo D-R respalda la preferencia de adsorción en microporos ${ }^{13}$ y asume una distribución de energía Gaussiana en una superficie heterogénea. El valor aparente de la energía de adsorción $\left(\mathrm{E}=19,61 \mathrm{KJ}_{\mathrm{mol}} \mathrm{m}^{-1}\right)$ hace referencia a la transferencia de energía libre de un mol de soluto desde el infinito (en solución) hacia la superficie del adsorbente ${ }^{13} \mathrm{y}$ al presentar un valor mayor a $8 \mathrm{KJ} \mathrm{mol}^{-1}$ da a entender que el proceso de adsorción es del tipo químico (quimisorción) ${ }^{13}$. Además la capacidad máxima de adsorción $\left(\mathrm{q}_{\mathrm{s}}=594,07 \mathrm{mg} \cdot \mathrm{g}^{-1}\right)$ predicha por el modelo D-R esta en concordancia con el rango experimental obtenido en esta investigación (entre 500 y $700 \mathrm{mg} \cdot \mathrm{g}^{-1}$ ).

Tabla 4. Parámetros y coeficientes de correlación para los modelos de isotermas de Langmuir, Freundlich, Temkin y Dubinin-Radushkevich.

\begin{tabular}{|c|c|c|c|c|}
\hline \multicolumn{5}{|c|}{ ISOTERMA DE LANGMUIR } \\
\hline $\mathrm{R}^{2}$ & $\mathrm{q}_{\max }\left(\mathrm{mg} \cdot \mathrm{g}^{-1}\right)$ & $\mathrm{K}_{\mathrm{L}}$ & $\left.g^{-1}\right)$ & $\chi^{2}$ \\
\hline 0,2614 & 0,552 & & & $1,66 \times 10^{3}$ \\
\hline \multicolumn{5}{|c|}{ ISOTERMA DE TEMKIN } \\
\hline $\mathrm{R}^{2}$ & $\mathrm{RT} / \mathrm{b}_{\mathrm{T}}\left(\mathrm{KJ} \cdot \mathrm{mol}^{-1}\right)$ & & & $\chi^{2}$ \\
\hline 0,8607 & 327,57 & & & 173,7 \\
\hline \multicolumn{5}{|c|}{ ISOTERMA DE FREUNDLICH } \\
\hline $\mathrm{R}^{2}$ & $\mathrm{n}_{\mathrm{F}}$ & $\mathrm{K}_{\mathrm{F}} \mathrm{mg} . \mathrm{g}$ & $\left.\mathrm{mg}^{-1}\right)^{1 / \mathrm{n}}$ & $\chi^{2}$ \\
\hline 0,7810 & 0,6437 & & & 820,1 \\
\hline \multicolumn{5}{|c|}{ ISOTERMA DE DUBININ-RADUSHKEVICH } \\
\hline $\mathrm{R}^{2}$ & $\mathrm{~B}_{\mathrm{DR}}$ & $\mathrm{E}\left(\mathrm{KJ} \cdot \mathrm{mol}^{-1}\right)$ & $\mathrm{q}_{\mathrm{s}}\left(\mathrm{mg} \cdot \mathrm{g}^{-1}\right)$ & $\chi^{2}$ \\
\hline 0,8904 & $2,60 \times 10^{-3}$ & 19,61 & 594,07 & 161,7 \\
\hline
\end{tabular}




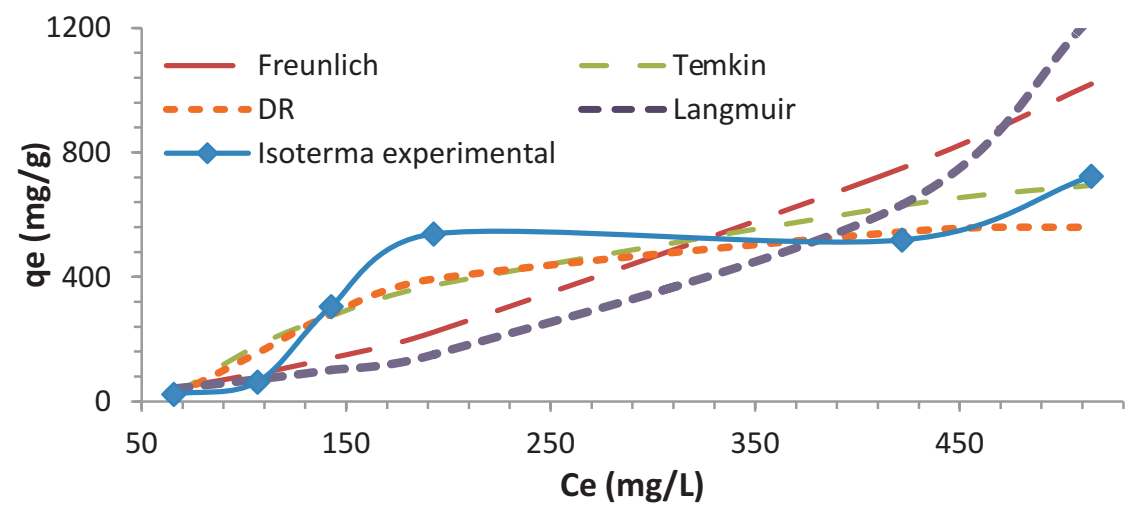

Figura 8. Comparación de la isoterma de adsorción experimental con los modelos de isotermas

Por otro lado, el modelo de Temkin asume que el calor de adsorción del adsorbato en una capa decrecerá linealmente al incrementar el recubrimiento de la superficie del adsorbente a concentraciones moderadas. El ajuste lineal permitió estimar el parámetro $\mathrm{RT} / \mathrm{b}_{\mathrm{T}}=327$ $\mathrm{KJ} \mathrm{mol}^{-1}$ (tabla 4), el cual se asocia al calor de adsorción, que al ser mayor a $200 \mathrm{KJ}^{\mathrm{mol}}{ }^{-1}$ indicaría que el proceso de adsorción era del tipo químico (quimisorción).

\section{CONCLUSIONES}

Se ha realizado la síntesis solvotérmica del MOF a partir de ácido fórmico y nitrato de magnesio hexahidratado en DMF a altas temperaturas $\left(96\right.$ a $\left.110^{\circ} \mathrm{C}\right)$ con rendimientos alrededor del $60 \%$. El análisis de la composición química realizada por EDX verificó la reproducibilidad del método de síntesis aplicado. Mediante el análisis por difracción de rayos $\mathrm{X}$ se identificó la fase cristalina del MOF como Dashkovaita. El análisis morfológico mostró que las partículas cristalinas del MOF presentaban una forma hexagonal y estaban formadas por filamentos de alrededor de 7,5 $\mu \mathrm{m}$ de longitud.

El proceso de adsorción, en general, es lento, con una alta capacidad de adsorción de DMA (qe $=307,96 \mathrm{mg} \cdot \mathrm{g}^{-1}$ a partir de una concentración inicial de $500 \mathrm{mg} \cdot \mathrm{g}^{-1}$ de DMA). Los resultados cinéticos se ajustaron mejor a los modelos de pseudo segundo orden y de Elovich, con una influencia importante de la difusión intraparticular. En el caso de la isoterma de adsorción, los mejores ajustes con la parte experimental se lograron con los modelos de Temkin y DubininRadushkevich, por medio de los cuales se pudo elucidar que el proceso de adsorción es del tipo químico sobre una superficie heterogénea.

\section{AGRADECIMIENTOS}

Este estudio fue financiado por el Proyecto No 401-2012-CONCYTEC-OAJ y el Proyecto DGI 2013-0016 concedido por el CONCYTEC y la Dirección de Gestión de la Investigación de la Pontificia Universidad Católica del Perú, respectivamente. Los autores también agradecen a los Drs. Nikolaos Kourkoumelis (Universidad de Loannina, Grecia) y Robert Papoular (CEA-Francia) por el análisis e interpretación de los DRX de los MOF, y a la Sección Química del Departamento de Ciencias de la PUCP por el apoyo brindado para el desarrollo de esta investigación. 


\section{BIBLIOGRAFÍA}

1. Yaghi, O. O'Keeffe, M.Ockwig, N. Chae, H. Eddaoudi, M. Kim, J. Reticular synthesis and the design of new materials. Nature. 2003; 423: 705-714.

2. Li, J. Sculley, J. Zhou, H. Metal-Organic Frameworks for Separations. Department of Chemistry, Texas A\&M University. Chemical Reviews. 2012; 112 (2): 869-932.

3. Helali, S. Puzenat, E. Perol, N. Safi, M. Guillard, C. Methylamine and dimethylamine photocatalytic degradation- Adsorption. Applied Catalysis A: General. 2011; 402: 201207.

4. Andrzejewski, P.Kasprzyk-Hordern, B.Nawrocki, J. N-nitrosodimethylamine (NDMA) formation during ozonation of dimethylamine-containing waters. Water research. 2008; 42: 863-870.

5. Límite máximo permisible de la dimetilamina. Disponible en:

http://69.175.53.20/msdshazcom/htdocs/workplace_chemicals/CAS 124-40-3.htm Fecha de consulta. 20 de febrero de 2014

6. Siddiqi, Z. Pathania, D. Rapid, selective and direct spectrophotometric determination of aliphatic amines with m-dinitrobenzene. Talanta. 2003; 60: 1197-1203.

7. Ho, Y. Selection of optimum sorption isotherm. Carbon. 2004; 42: 2113-2130.

8. Rood, J.A. Noll, B. Henderson, K.W. Synthesis, Structural Characterization, Gas Sorption and Guest-Exchange Studies of the Lightweight, Porous Metal- Organic Framework $\alpha-\mathrm{Mg}_{3}\left(\mathrm{O}_{2} \mathrm{CH}\right)_{6}$. Inorganic chemistry. 2006; 45 (14): 5521-5528.

9. Ficha JCPDS 53-1187 correspondiente a la Dashkovaite. Disponible en: http://www.icdd.com/products/ Fecha de consulta: 23 de febrero de 2014

10. Giles, C. Smith, D. Huitson, A. A general treatment and classification of the solute adsorption isotherm. I. Theoretical. Journal of Colloid and Interface Science. 1974; 47 (3): 755-765.

11. Tan, I.A.W. Ahmad, A.L. Hameed, B.H. Adsorption isotherms, kinetics, thermodynamics and desorption studies of 2, 4, 6-trichlorophenol on oil palm empty fruit bunch-based activated carbon. Journal of Hazardous Materials. 2009; 164: 473-482.

12. Ho, Y. S. McKay, G. Sorption of copper (ii) from aqueous solution by peat. Water, Air, and Soil Pollution. 2004; 158: 77-97.

13. Chen, A. Chen, S. Biosorption of azo dyes from aqueous solution by glutaraldehydecrosslinked chitosans. Journal of Hazardous Materials. 2009; 172: 1111-1121. 